\title{
The Value of Ultrasound-Guided Fine-Needle Aspiration Cytology Combined with Puncture Feeling in the Diagnosis of Thyroid Nodules
}

\author{
Li Li $^{a, b}$ Xiao Chen ${ }^{b}$ Peipei Li ${ }^{b}$ Yibo Liua,b Xiaoli Mac Yu-Quan Ye ${ }^{a, b}$ \\ aSchool of Graduate, Hebei Medical University, Shijiazhuang, China; ${ }^{\text {b}}$ Department of Ultrasound, Hebei General \\ Hospital, Shijiazhuang, China; 'Department of Pathology, Hebei General Hospital, Shijiazhuang, China
}

\section{Keywords}

Puncture feeling - Benign nodules · Malignant nodules · Ultrasound-guided fine-needle aspiration cytology · Thyroid nodules

\begin{abstract}
Introduction: There are few studies on the role of puncture feeling in thyroid nodules during ultrasound-guided fineneedle aspiration cytology (US-FNAC), although it is expected to become a new predictive technique. We aimed to analyze the importance of puncture feeling in combination with US-FNAC and investigate whether it can be used as an indicator to predict the nature of thyroid nodules. Materials and Methods: From January 1, 2018, to October 31, 2020, a total of 623 thyroid nodules were included. Puncture feeling was classified as "soft," "hard," or "hard with grittiness." The correlation between puncture feeling and postoperative pathology and the diagnostic value of FNAC combined with puncture feeling were analyzed, and the influence of thyroid nodule size on puncture feeling, FNAC, and FNAC combined with puncture feeling was studied. We further explored the correlation between puncture feeling and histopathology in Bethesda III and IV thyroid nodules. Results: There was a significant correlation between puncture feeling and postoperative pathology $(p<0.001)$. The sensitivity, negative predictive value, and total coincidence rate of US-FNAC combined with puncture feeling for the diagnosis of thyroid nodules were higher than those of US-FNAC alone (96.1,
\end{abstract}

83.6 , and $94.7 \%$ vs. $89.0,65.5$, and $89.7 \%$, respectively). Thyroid nodule size was the influencing factor for puncture feeling, FNAC, and FNAC combined with puncture feeling ( $p<$ 0.05 for all). The area under the curve for puncture feeling, FNAC, and FNAC combined with puncture feeling for thyroid nodules of size $\leq 1 \mathrm{~cm}$ was greater than for modules of size $>1 \mathrm{~cm}$. Puncture feeling was of great value in diagnosing Bethesda III thyroid nodules ( $p<0.001$ ), and all Bethesda IV thyroid nodules had puncture feeling of soft. Conclusion: Puncture feeling is of great value during US-FNAC. "Hard" and "hard with grittiness" were indicators for malignancy, while "soft" indicated that the thyroid nodule was likely to be benign. The diagnostic value of US-FNAC combined with puncture feeling for thyroid nodules is higher than US-FNAC alone, especially when nodule size is $\leq 1 \mathrm{~cm}$. Puncture feeling is of great value in predicting the nature of Bethesda III thyroid nodules.

(c) 2021 The Author(s)
Published by S. Karger AG, Basel

\section{Introduction}

The incidence of thyroid nodules in the general population is on the rise. It is estimated that $4-7 \%$ are detected by palpation and $10-60 \%$ are detected by ultrasound screening [1-4]. Ultrasound-guided (US) fine-needle aspiration (FNA) cytology (FNAC) has become a diagnostic method for high-risk thyroid nodules. It has high sensitivity and specificity, but it still misses the diagnosis of
(C) 2021 The Author(s)

Published by S. Karger AG, Basel

This is an Open Access article licensed under the Creative Commons Attribution-NonCommercial-4.0 International License (CC BY-NC) (http://www.karger.com/Services/OpenAccessLicense), applicable to the online version of the article only. Usage and distribution for commercial purposes requires written permission.
Correspondence to:

Yu-Quan Ye, wangzhichao910920@163.com 
Fig. 1. Flow diagram of thyroid nodules included in the study. FNAC, fine-needle aspiration cytology.

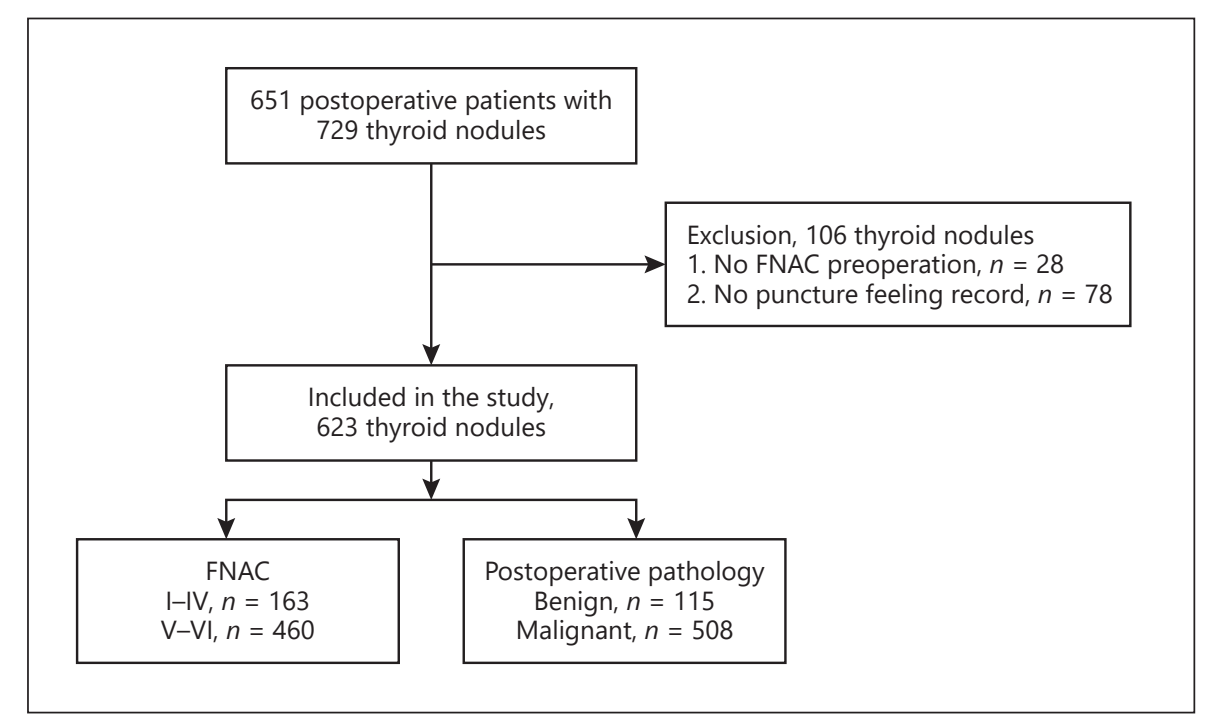

some malignant thyroid nodules. The diagnostic efficiency of FNAC is affected by many factors; it is not known whether the size of thyroid nodules affects the results of FNAC [5].

Luo et al. [6] have shown that a puncture feeling of grittiness is an indicator of malignant thyroid nodules; a puncture feeling of grittiness combined with FNAC can significantly improve the accuracy of diagnosis of malignant thyroid nodules. We have observed during FNAC that when the needle tip passes through different tissues, the operator will sense different puncture feelings. Different thyroid nodules provide different puncture feelings, such as "soft," "hard," and "hard with grittiness." Although Luo et al. [6] studied the predictive value of a puncture feeling of grittiness in thyroid nodules, they did not classify needle sensation in detail. Thyroid nodules with a puncture feeling of "hard without grittiness" are also worth exploring.

To date, there are few studies on puncture feeling. Therefore, this study aimed to analyze the correlation between puncture feeling and postoperative pathology, assess the value of US-FNAC combined with puncture feeling for diagnosis of thyroid nodules, and study the influence of thyroid nodule size on puncture feeling, FNAC, and FNAC combined with puncture feeling.

\section{Materials and Methods}

\section{Patients}

This study is a retrospective study. The histopathological results of all patients were judged jointly by 3 professional pathologists. We obtained the clinicopathological information data of all patients by consulting the patients' medical records data. From January 1, 2018, to October 31, 2020, there were 729 thyroid nodules in 651 patients, of which 106 were excluded ( 28 had no preoperative FNAC and 78 had no puncture feeling recorded). Finally, 623 thyroid nodules with preoperative FNAC and puncture feeling records were included in the study. The detailed selection process is shown in Figure 1.

\section{Ultrasound-Guided FNA Procedure}

All FNA procedures were performed by the same experienced doctor. All patients were examined using the Philips EPIQ 7 (Philips, Healthcare, Cambridge, MA) color Doppler ultrasound system, using a linear array probe at a frequency of 5-12 MHz. The puncture needle was a $23 \mathrm{G}$ or $25 \mathrm{G}$ needle (Hakko, Chikuma, Japan). Before FNA, ultrasonography was used to evaluate and locate thyroid nodules. The location, size, shape, aspect ratio, boundary, echo level, calcification, blood supply, posterior echo, and relationship with surrounding tissues were recorded. At the same time, the operator evaluated whether there was lymph node metastasis. The surgeon confirmed the puncture site and puncture path, placed the patient in a supine position, disinfected, spread aseptic hole towels, and used lidocaine for local infiltration anesthesia. Using ultrasound guidance, the operator performed FNA biopsy; the needle was inserted into the nodule and moved back and forth for approximately 5-10 passes in different parts of the nodule to ensure adequate sampling. Then, the aspiration sample was quickly smeared onto a slide and fixed with 95\% ethyl alcohol. Thyroid nodules were sampled 2-4 times and then sent to the Pathology Department for cytological diagnosis by 2 professional pathologists. The operator informed the recorder of the puncture feeling during the FNA process. Puncture feeling was defined as the texture of thyroid nodules felt by the operator during the process, which was divided into 3 categories, as follows: 0, "soft" without any resistance; 1, "hard" and with any degree of displacement of the nodule during the FNA process; and 2, "hard with grittiness" and with any degree of displacement of the nodule during the FNA process. 


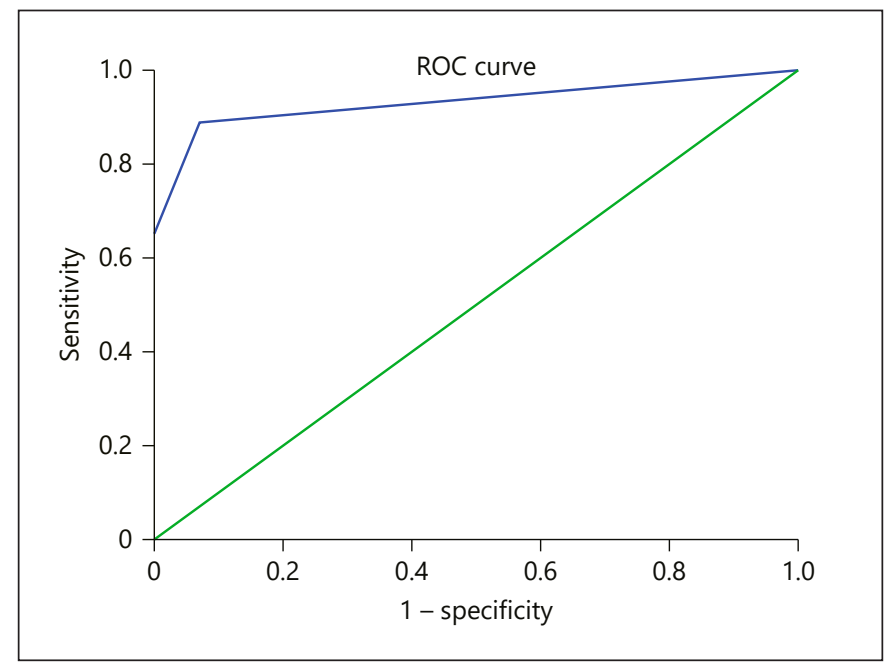

Fig. 2. Correlation between Bethesda classification and histopathology. ROC, receiver operating characteristic.

\section{Criteria for Classification}

FNAC is classified according to the Bethesda System for Reporting Thyroid Cytopathology, as follows: I, nondiagnostic or unsatisfactory; II, benign; III, atypia of undetermined significance or follicular lesion of undetermined significance; IV, follicular neoplasm or suspicious for follicular neoplasm; V, suspicious for malignancy; and VI, malignant. According to the Bethesda System, V and VI are classified as malignant, while I-IV are considered benign.

In diagnosis using puncture feeling combined with FNAC, if puncture feeling is "hard" or "hard with grittiness," thyroid nodules of Bethesda III-VI are diagnosed as malignant; if puncture feeling is "soft," thyroid nodules of Bethesda II-IV are diagnosed as benign. In other cases, FNAC prevails.

\section{Statistical Analysis}

Statistical analysis was performed using SPSS 19.0 (IBM, Armonk, NY). Comparisons of categorical variables were performed using the $\chi^{2}$ test and Fisher's exact test with $95 \%$ confidence interval (CI). Taking postoperative pathological results as the standard, a receiver operating characteristic (ROC) curve was constructed. The area under the curve (AUC) was calculated, and the sensitivity, specificity, positive predictive value, negative predictive value, and total consistent rate of FNAC and of FNAC combined with puncture feeling were calculated. $p$ values of $<0.05$ were considered statistically significant.

\section{Results}

A total of 623 thyroid nodules were included in the study, of which 115 were benign, including 81 nodular goiter, 15 follicular thyroid adenoma, 10 subacute thyroiditis, and 9 Hashimoto. There were 508 malignant thyroid nodules, including 501 papillary thyroid carcinoma,
Table 1. Clinicopathological characteristics of patients with thyroid nodules

\begin{tabular}{lllc}
\hline & \multirow{2}{*}{ Total } & \multicolumn{2}{l}{ Postoperative pathology } \\
\cline { 3 - 4 } & & benign & malignant \\
\hline Age, years & $47.3(7-88)$ & $51.6(22-74)$ & $46.5(7-88)$ \\
$\begin{array}{l}\text { Gender, } n(\%) \\
\quad \text { Male }\end{array}$ & $135(21.7)$ & $35(30.4)$ & $100(19.7)$ \\
$\quad$ Female & $488(78.3)$ & $80(69.6)$ & $408(80.3)$ \\
FNA pathology, $n(\%)$ & & & \\
$\quad$ Benign & $163(26.2)$ & $107(93.0)$ & $56(11.0)$ \\
$\quad$ Malignant & $460(73.8)$ & $8(7.0)$ & $452(89.0)$ \\
$\begin{array}{l}\text { Puncture feeling, } n \\
\text { (\%) }\end{array}$ & & & \\
$\quad 0$ & $92(14.8)$ & $71(61.7)$ & $21(4.1)$ \\
1 & $412(66.1)$ & $40(34.8)$ & $372(73.2)$ \\
2 & $119(19.1)$ & $4(3.5)$ & $115(22.7)$ \\
Size, $n(\%)$ & $393(63.1)$ & $49(42.6)$ & $344(67.7)$ \\
$\quad \leq 1 \mathrm{~cm}$ & $230(36.9)$ & $66(57.4)$ & $164(32.3)$ \\
\hline $1 \mathrm{~cm}$ & 623 & $115(18.5)$ & $508(81.5)$ \\
\hline Total & & &
\end{tabular}

FNA, fine-needle aspiration.

4 follicular thyroid carcinoma, 2 medullary carcinoma, and 1 anaplastic carcinoma. According to the Bethesda system, there were 163 thyroid nodules classified into categories I-IV. Category I did not include any nodules. Category II included 51 nodular goiters, 6 Hashimoto's thyroiditis, and 5 subacute thyroiditis. Among all FNAC categories, category III accounted for 90 nodules, and category IV included 11 follicular tumors. There were 460 thyroid nodules in categories V-VI, including 130 suspicious for malignant and 330 papillary carcinomas. The pathological classifications of all 623 thyroid nodules are shown in Figure 1. With histological pathology as the gold standard, we calculated the ROC curve of the diagnostic value of Bethesda categorization (i.e., cytological pathology) for thyroid nodules, as shown in Figure 2. The results showed a significant correlation between Bethesda classification and histopathology $(p<0.001)$. The AUC was 0.933 ; 95\% CI: 0.914-0.953.

The clinicopathological characteristics of the 623 thyroid nodules included in this study are shown in Table 1. The average age of all patients was $47.3(7-88)$ years. The majority of patients were female $(488$ [78.3\%] vs. 135 [21.7\%]). In this study, most thyroid nodules were $\leq 1 \mathrm{~cm}$ in size (393 [63.1\%] vs. 230 [36.9\%]). Puncture feeling, which was divided into 3 categories, was as follows: category 0: 92 (14.8\%), category 1: 412 (66.1\%), and catego- 


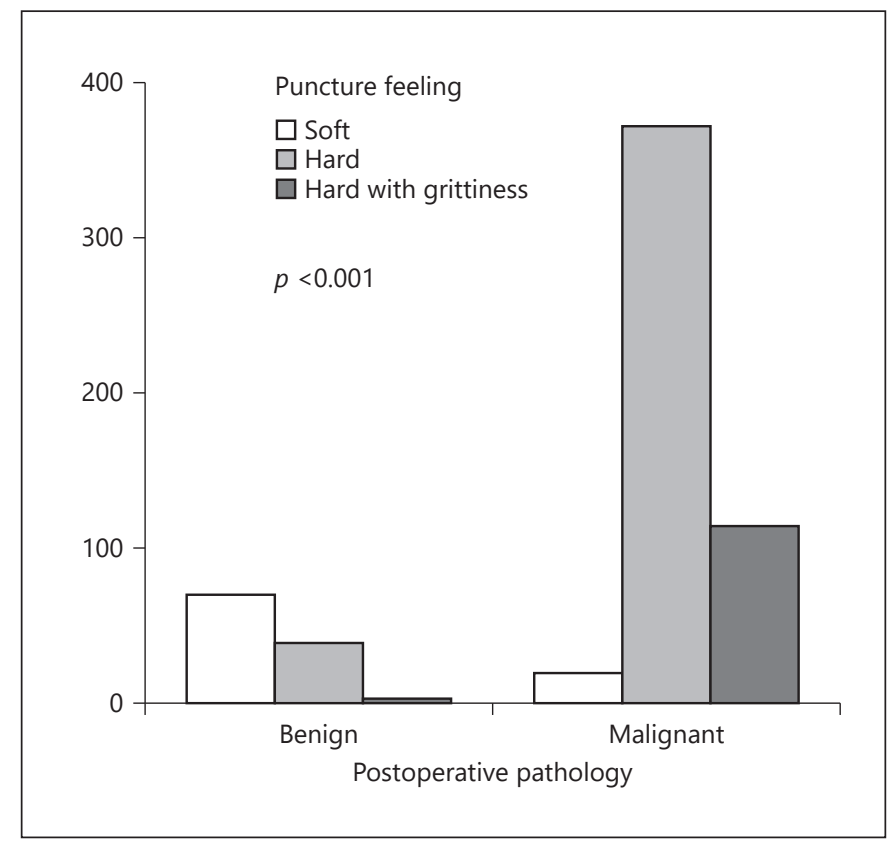

Fig. 3. Correlation between puncture feeling and postoperative pathology.

ry 2: 119 (19.1\%). The puncture feelings of "hard" and "hard with grittiness" identified up to 95.9\% (487/508) of malignant nodules, while the feeling "soft" identified $61.7 \%$ (71/115) benign nodules. For the 3 categories of puncture feeling, category 0 identified $77.2 \%$ (71/92) of benign nodules, category 1 identified 90.3\% (372/412) of malignant nodules, and category 2 identified $96.6 \%$ $(115 / 119)$ of malignant nodules. The analysis results reveal that there was a significant correlation between puncture feeling and postoperative pathology $(p<0.001)$ (Fig. 3). The above results show that the puncture feelings "hard" and "hard with grittiness" are indicators of malignant thyroid nodules. Especially thyroid nodules with the puncture feeling of "hard with grittiness" were more likely to be malignant. The puncture feeling of "soft" tended to suggest that thyroid nodules were benign.

We analyzed the diagnostic value for thyroid nodules of FNAC and FNAC combined with puncture feeling (Table 2). The sensitivity, specificity, positive predictive value, negative predictive value, and total coincidence rate for FNAC were 89.0, 93.0, 98.3, 65.5, and 89.7\%, respectively. For FNAC combined with puncture feeling, the corresponding values were 96.1, 88.7, 97.4, 83.6, and $94.7 \%$, respectively. The sensitivity, negative predictive value, and total coincidence rate of FNAC combined with puncture feeling were higher than those of FNAC. How- ever, the specificity and positive predictive value of FNAC combined with puncture feeling were slightly lower than those of FNAC, which may be due to some benign thyroid nodules having the puncture feeling of "hard" or "hard with grittiness," for which they were classified as malignant.

In Table 3, it is shown that the size of thyroid nodules was the influencing factor for FNAC, puncture feeling, and FNAC combined with puncture feeling, with $p$ values of $<0.001,0.001$, and $<0.001$, respectively. According to the size of thyroid nodules ( $\leq 1$ and $>1 \mathrm{~cm}$ ), 623 nodules were divided into 2 groups, and then the ROC curves were analyzed for FNAC, puncture feeling, and FNAC combined with puncture feeling, according to the postoperative pathology (Fig. 4). The results show that the AUC of FNAC combined with puncture feeling $(0.930,95 \% \mathrm{CI}$ : $0.876-0.984)$ was greater than those of FNAC and puncture feeling alone $(0.910,95 \%$ CI: $0.862-0.958$, and 0.832 , $95 \%$ CI: $0.758-0.906$, respectively) when the size of the thyroid nodule was $\leq 1 \mathrm{~cm}(p<0.001$ for all). When the nodule size was $>1 \mathrm{~cm}$, the AUC of FNAC combined with puncture feeling (0.904, 95\% CI: 0.855-0.954) was also higher than those of FNAC and puncture feeling alone (0.903, 95\% CI: $0.857-0.948$, and 0.793, 95\% CI: $0.724-$ 0.861 , respectively) ( $p<0.001$ for all). However, when nodule size was $>1 \mathrm{~cm}$, the corresponding AUC values all reduced, which showed that FNAC, puncture feeling, and FNAC combined with puncture feeling were more accurate when the size of the thyroid nodule was $\leq 1 \mathrm{~cm}$.

In this study, $14.4 \%(90 / 623)$ of thyroid nodules were classified as Bethesda III after FNAC. We further analyzed the correlation between puncture feeling and postoperative pathology of Bethesda III thyroid nodules. The results show that puncture feeling was of great value in diagnosing Bethesda III thyroid nodules $(p<0.001)$ (Table 4). Of all the Bethesda III nodules, 34.4\% (31/90) were malignant and 65.6\% (59/90) were benign. For "soft" puncture feeling, $95.9 \%$ (47/49) of thyroid nodules were benign. For "hard" puncture feeling, 58.6\% (17/29) of thyroid nodules were malignant. For "hard with grittiness" puncture feeling, $100.0 \%$ (12/12) of thyroid nodules were malignant. This proved that puncture feeling had important predictive value for the nature of Bethesda III thyroid nodules. "Hard" and "hard with grittiness" were indicators for malignancy, while "soft" may be a predictor of benign nodules. However, $41.4 \%$ of the thyroid nodules with "hard" puncture feeling were benign; these may be distinguished from malignant nodules using ultrasound image features to avoid excessive diagnosis and treatment. In this study, only 11 Bethesda IV thyroid nod- 
Table 2. Diagnostic value of FNA and FNA combined with puncture feeling in thyroid nodules

\begin{tabular}{|c|c|c|c|c|c|c|c|c|}
\hline Diagnostic method & & \multicolumn{2}{|c|}{ Postoperative pathology } & $\begin{array}{l}\text { Sensitivity, } \\
\%\end{array}$ & $\begin{array}{l}\text { Specificity, } \\
\%\end{array}$ & $\begin{array}{l}\mathrm{PV}+, \\
\%\end{array}$ & $\begin{array}{l}\mathrm{PV}-\text {, } \\
\%\end{array}$ & $\begin{array}{l}\pi \\
\%\end{array}$ \\
\hline $\begin{array}{l}\text { FNA combined with } \\
\text { puncture feeling }\end{array}$ & $\begin{array}{l}\text { Benign } \\
\text { Malignant }\end{array}$ & $\begin{array}{r}102 \\
13\end{array}$ & $\begin{array}{r}20 \\
488\end{array}$ & 96.1 & 88.7 & 97.4 & 83.6 & 94.7 \\
\hline
\end{tabular}

FNA, fine-needle aspiration; $\mathrm{PV}+$, positive predictive value; $\mathrm{PV}-$, negative predictive value; $\pi$, total consistent rate.

Table 3. Effect of thyroid nodule size on FNA, puncture feeling, and FNA combined puncture feeling

\begin{tabular}{|c|c|c|c|c|}
\hline \multirow[t]{2}{*}{ Diagnostic method } & & \multicolumn{2}{|l|}{ Size } & \multirow[t]{2}{*}{$p$ value } \\
\hline & & $\leq 1 \mathrm{~cm}$ & $>1 \mathrm{~cm}$ & \\
\hline \multirow[t]{2}{*}{ FNA } & Benign & 79 & 84 & \multirow{2}{*}{$<0.001$} \\
\hline & Malignant & 314 & 146 & \\
\hline \multirow[t]{3}{*}{ Puncture feeling } & Soft & 42 & 50 & \multirow{3}{*}{0.001} \\
\hline & Hard & 270 & 142 & \\
\hline & Hard with grittiness & 81 & 38 & \\
\hline \multirow[t]{2}{*}{ FNA combined with puncture feeling } & Benign & 49 & 73 & \multirow{2}{*}{$<0.001$} \\
\hline & Malignant & 344 & 157 & \\
\hline
\end{tabular}

FNA, fine-needle aspiration.

ules were included. All of these had "soft" puncture feeling, independent of histological pathology.

In Figures 5 and 6, we show 2 cases with Bethesda III cytology with different histopathology and puncture feeling. Figure 5 is a case of papillary thyroid carcinoma where puncture feeling was "hard." Figure 6 is a case of hyperplasia of thyroid follicular cells where puncture feeling was "soft."

\section{Discussion}

FNAC plays an important role in the diagnosis of thyroid nodules. Its purpose is to prevent unnecessary operations for benign lesions and avoid misdiagnosis of malignant lesions, as far as possible [7]. The challenge for endocrinologists, surgeons, and pathologists is to achieve an accurate preoperative diagnosis of malignant tumors to ensure that patients receive appropriate treatment [8]. Although FNAC has high sensitivity and specificity, there is still a possibility of false positive and false negative results.
During the FNA procedure, the operator can feel differences in stiffness of skin, muscle, and lesions. Recently, a study by Luo et al. [6] showed for the first time that there is a correlation between the puncture feeling of "grittiness" and malignant thyroid lesions. Our results proved that there was a significant correlation between puncture feeling and postoperative pathology $(p<0.001)$ (Fig. 3). "Hard" and "hard with grittiness" puncture feelings are indicators for malignancy, and "soft" puncture feeling is more likely to indicate a benign nodule. In this study, the sensitivity, negative predictive value, and total coincidence rate of FNAC combined with puncture feeling were significantly higher than those of FNAC alone. In short, compared with FNAC, FNAC combined with puncture feeling reduced uncertain diagnosis and improved diagnostic sensitivity.

The reason why puncture feeling during the US-FNAC process is related to postoperative pathology is the stiffness of the lesion, determined by the structural characteristics of its internal components. Nodular goiter and follicular adenoma are common in benign thyroid nodules. 
Table 4. Correlation between puncture feeling and postoperative pathology in Bethesda III thyroid nodules

\begin{tabular}{lllll}
\hline Puncture feeling & Total, $n(\%)$ & \multicolumn{2}{l}{ Postoperative pathology } & $p$ value \\
\cline { 3 - 4 } & & malignant, $n(\%)$ & benign, $n(\%)$ & \\
\hline Soft & $49(54.5)$ & $2(4.1)$ & $47(95.9)$ & \\
Hard & $29(32.2)$ & $17(58.6)$ & $12(41.4)$ & $<0.001$ \\
Hard with grittiness & $12(13.3)$ & $12(100.0)$ & $59(6)$ & \\
\hline Total & $90(100.0)$ & $31(34.4)$ & $59.6)$ & \\
\hline
\end{tabular}

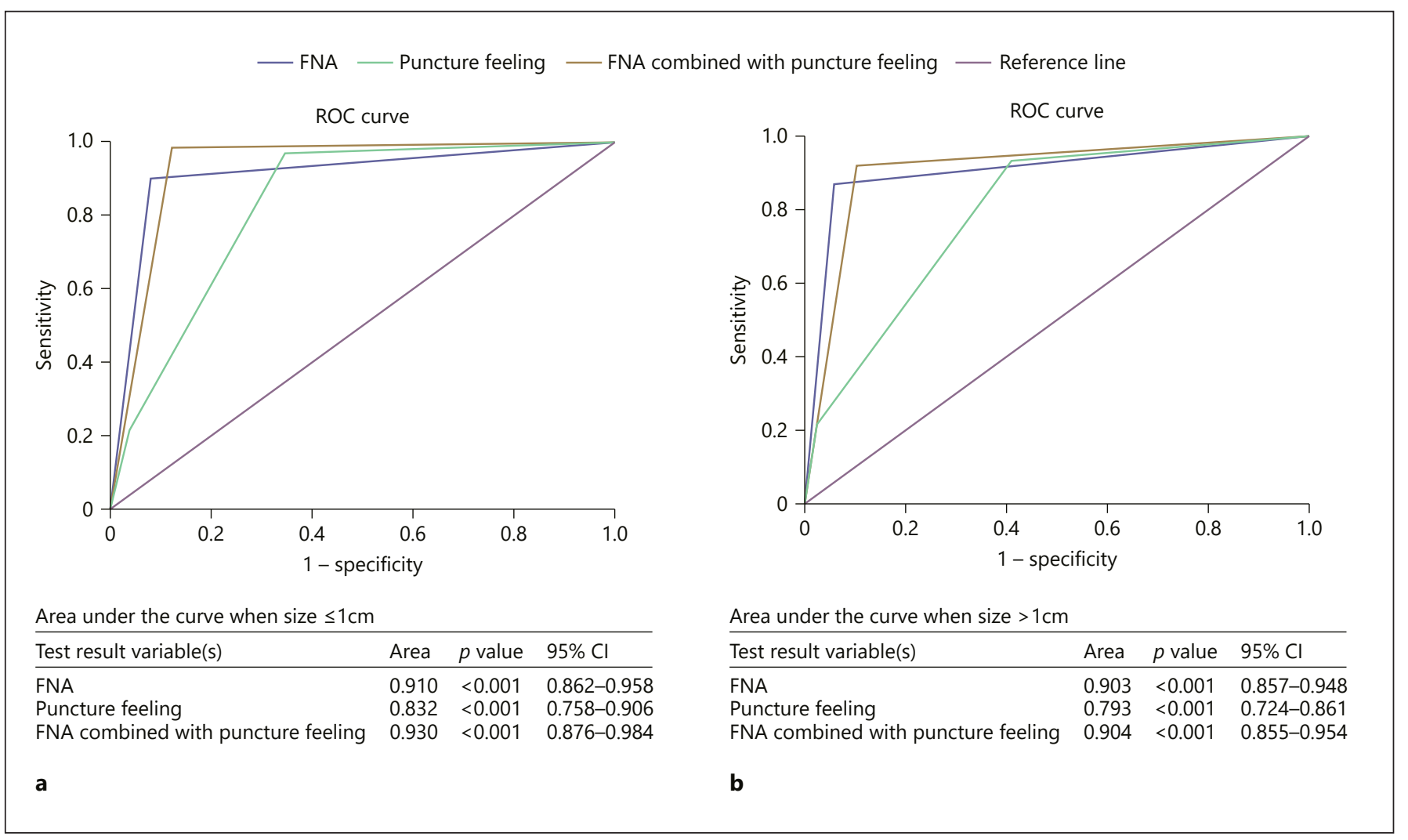

Fig. 4. The ROC curve of FNA, puncture feeling, and puncture feeling combined with FNA when sizes are $\leq 1 \mathrm{~cm}$ and $>1 \mathrm{~cm}$. ROC, receiver operator characteristic; FNA, fine-needle aspiration.

Nodular goiter is composed of follicles with different degrees of expansion, and the cavity is full of colloid. Follicular adenoma is composed of proliferating follicular epithelium with a large number of follicles and mostly edema-like loose fibrous interstitium. In these cases, the needle tip can smoothly enter the nodule without any resistance. If there is a tumor or inflammation in the lesion, its composition and structure change, and stiffness is increased [9]. Adenomatous goiter, a proliferative follicular lesion, is the most common neoplastic lesion of the thy- roid; follicular hyperplasia and degeneration occur alternately [10]. In patients with Hashimoto's thyroiditis, the stiffness of the thyroid parenchyma increases [11]. Thus, the puncture feeling in these patients may be "hard." A previous study has shown that although subacute thyroiditis is a self-limiting disease, the hardness of the hypoechoic area is greater than that of Hashimoto's thyroiditis [12]. Therefore, for most benign nodules, puncture feeling can be "soft"; when fibrosis or calcification occurs inside the nodules, puncture feeling can be "hard" or 

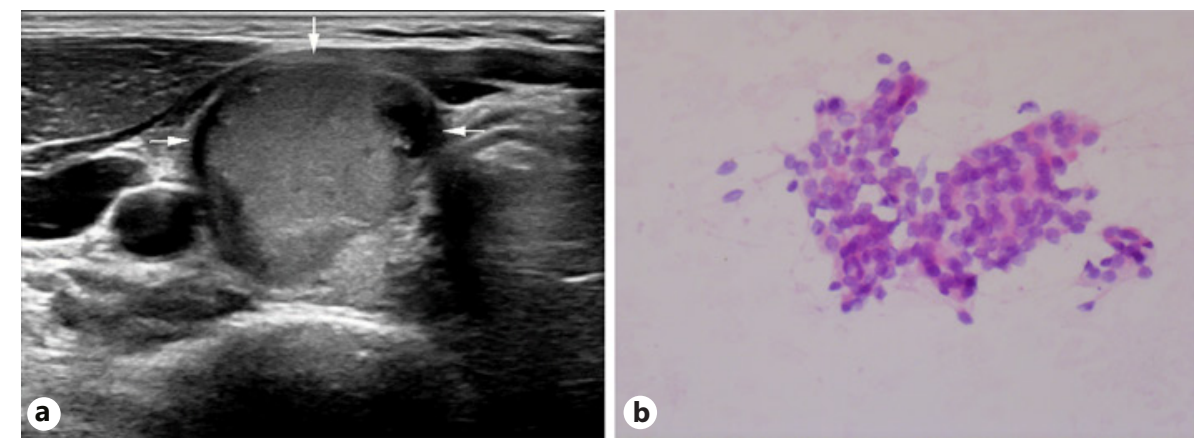

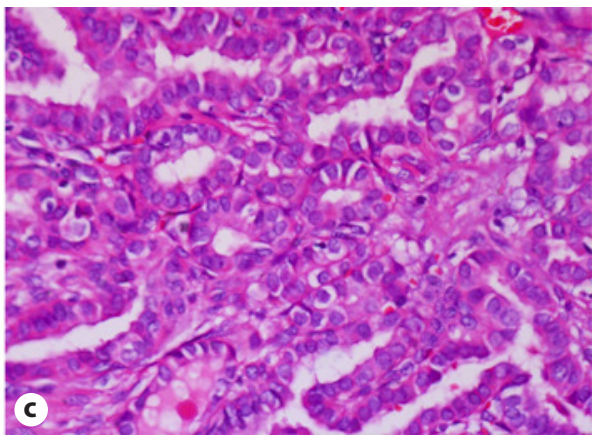

clear features, but the number of tumor cells was reduced (HE. $\times 400$ ). c Histopathology showed apparent papillary structure throughout the tumor. The nuclei of the cells of PTC could be grouped into 3 categories: (1) enlargement, overlapping, and nuclear elongation; (2) irregularities of the membranes; and (3) pale nuclei with powdery chromatin (HE. $\times 400)$.
Fig. 5. Image of a 48-year-old woman with a thyroid nodule in the right lobe whose puncture feeling was "hard." a The ultrasound image showed that the thyroid nodule had a clear boundary, uneven internal echo, microcalcification, partial liquefaction, and aspect ratio $>1$. $\mathbf{b}$ The cytological pathology was Bethesda III category, which showed that the cells were composed of monolayer sheets with a syncytial-like appearance and thyroid carcinoma nu-
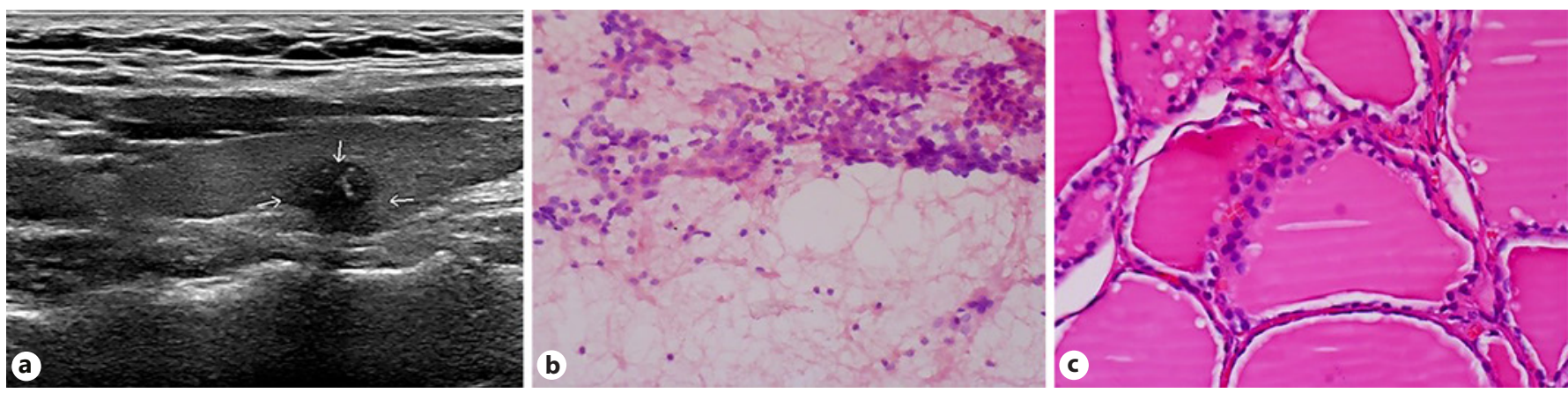

Fig. 6. Image of a 55-year-old man with a thyroid nodule in the left lobe whose puncture feeling was "soft." a The ultrasound image showed unclear boundary, irregular shape, uneven echo, and microcalcification of the thyroid nodule. $\mathbf{b}$ The cytological pathology was the Bethesda III category, which showed that the cells were composed of monolayer sheets with a syncytial-like appearance, but the thyroid carcinoma nuclear features were insufficient (HE. $\times 400$ ). c Histopathology showed thyroid follicular hyperplasia, which forms a normal follicular structure (HE. $\times 400)$. "hard with grittiness." This can lead to false positive results, but the surgeon can combine the characteristics of the ultrasound image of the thyroid nodule, clinical manifestations, and laboratory tests to eliminate some false positive results.

Papillary carcinoma is the most common type of thyroid cancer. There are relatively few follicles and glial components in papillary thyroid carcinoma tissue. Due to slow growth, psammoma bodies, which are the product of a bioactive process, can be found in most lesions. Microcalcification is formed by irregular calcium salt deposition, which manifests as sand-gravel accumulation. A puncture feeling of "grittiness" is probably due to microcalcification. Calcification is common in thyroid nodules, with the type and incidence of calcification varying in different lesions $[13,14]$. Coarse calcification is a degenerative disease caused by excessive proliferation or rapid growth of cell tissue, which occurs in benign nodules. Microcalcification has been shown to be an important feature for distinguishing between benign and malignant thyroid nodules [15-17]. Calcifications inside the lesion can alter the stiffness of the lesion, as can peripheral calcifications $[18,19]$. Therefore, FNAC combined with a puncture feeling of "hard" and "hard with grittiness" can significantly improve sensitivity in diagnosis of thyroid nodules.

At present, there is no unified conclusion on the diagnostic efficiency of US-FNAC, especially when the nod- 
ules are too small or too large [20]. A previous study reported that the false negative rate for large nodules was higher, especially for those with a maximum diameter $>40.0 \mathrm{~mm}$ [21]. Leenhardt et al. [22] reported that diagnostic efficiency for lesions with a maximum diameter $\leq 10.0 \mathrm{~mm}$ was lower than for lesions with a maximum diameter $>10.0 \mathrm{~mm}$. However, Lyu et al. [23] reported that nodule size did not affect the diagnostic efficiency of USFNAC. Our study has proved that there is a significant correlation between the size of thyroid nodules and diagnosis using FNAC $(p<0.01)$, puncture feeling $(p<0.001)$, and FNAC combined with puncture $(p<0.001)$. The ROC curve further revealed that the AUCs of FNA, puncture feeling, and puncture feeling combined with FNAC for nodule sizes $\leq 1 \mathrm{~cm}$ were greater than for nodule sizes $>1$ $\mathrm{cm}$. The reason may be that when the size is $>1 \mathrm{~cm}$, the tissue is unevenly distributed in the nodule, or that the nodule can be accompanied by necrosis and liquefaction; therefore, it is easy to penetrate the necrotic part during puncture, which leads to a great difference in evaluations of the same category of nodule using puncture feeling.

At present, the common methods for the treatment of Bethesda III thyroid nodules are follow-up, repeated FNA, operation, molecular detection, and so on. However, Bethesda III thyroid nodules also have a malignancy risk, so a method should be found to screen for cases that should go to surgery and for which clinical and radiological/cytological follow-up is desirable. This study has shown that puncture feeling has a good predictive value for the nature of Bethesda III thyroid nodules (Fig. 4). If the puncture feeling of FNAC in Bethesda III thyroid nodules is "hard with grittiness," the case should go to surgery because the patient has a high risk of malignancy. "Hard" puncture feeling also has a relatively high malignancy risk. However, Hashimoto's thyroiditis and subthyroiditis increase stiffness, so when a "hard" puncture feeling is encountered with Bethesda III thyroid nodules, it is sometimes impossible to distinguish inflammatory lesions from malignant nodules. Therefore, repeated FNAC is needed to further confirm the diagnosis. In this study, when the puncture feeling of Bethesda III thyroid nodules was "soft," $95.9 \%$ of nodules were benign. Provided that puncture feeling is combined with ultrasound images to eliminate the risk of malignancy, clinical and radiological follow-up should be performed. The puncture feeling of 11 Bethesda IV thyroid nodules in this study was "soft," which was related to the internal components of follicular tumors. Follicular tumor originates from the follicular epithelium, including follicular adenoma and follicular carcinoma; the internal components are mainly follicular and glial, so the needle tip has no resistance to enter the follicular tumor, resulting in a "soft" puncture feeling. However, the number of Bethesda IV cases in this study is insufficient; more cases need to be further studied.

In conclusion, there is a significant correlation between puncture feeling and postoperative pathology. If puncture feeling is "soft," it indicates that the nodule may be benign. If puncture feeling is "hard" or "hard with grittiness," the nodule is likely to be malignant. This is more accurate in thyroid nodules with size $\leq 1 \mathrm{~cm}$. The results of this study prove that the combination of FNAC and puncture feeling significantly improves the diagnostic sensitivity of FNAC. In this study, puncture feeling was shown to be most valuable for the diagnosis of Bethesda III thyroid nodules. Puncture feelings of "hard" or "hard with grittiness" help us to distinguish malignant cases. "Soft" puncture feeling suggests that these cases can be followed up to avoid excessive diagnosis and treatment.

\section{Statement of Ethics}

Our research complied with the guidelines for human studies, and the research was conducted ethically in accordance with the World Medical Association Declaration of Helsinki. Ethical approval was obtained from the Medical Ethics Committee of Hebei General Hospital, Ethical Review No. 2021, Scientific Research Ethics Review No. 12. Before FNA, all patients signed informed consent for special treatment.

\section{Conflict of Interest Statement}

The authors declare that they have no conflicts of interest to this work.

\section{Funding Sources}

The study required no funding, and therefore it did not receive any forms of financial support.

\section{Author Contributions}

Yu-Quan Ye and Li Li designed the experiments; Xiaoli Ma and Yibo Liu collected and sorted all data; Xiao Chen and Peipei Li analyzed the data and drew tables and graphs; Li Li wrote the manuscript.

\section{Data Availability Statement}

As the database included in this study is deidentified, it is not available in an open access repository. Supporting data can be requested by emailing the corresponding author. 


\section{References}

1 Banks ND, Kowalski J, Tsai HL, Somervell H, Tufano R, Dackiw AP, et al. A diagnostic predictor model for indeterminate or suspicious thyroid FNA samples. Thyroid. 2008 Sep; 18(9):933-41.

2 Frates MC, Benson CB, Doubilet PM, Kunreuther E, Contreras M, Cibas ES, et al. Prevalence and distribution of carcinoma in patients with solitary and multiple thyroid nodules on sonography. J Clin Endocrinol Metab. 2006 Sep;91(9):3411-7.

3 Gharib H, Papini E, Valcavi R, Baskin HJ, Crescenzi A, Dottorini ME, et al. American Association of Clinical Endocrinologists and Associazione Medici Endocrinologi medical guidelines for clinical practice for the diagnosis and management of thyroid nodules. Endocr Pract. 2006 Jan-Feb;12(1):63-102.

4 Cooper DS, Cooper DS, Doherty GM, Haugen BR, Hauger BR, Kloos RT, et al. Revised American Thyroid Association management guidelines for patients with thyroid nodules and differentiated thyroid cancer. Thyroid. 2009 Nov; 19(11):1167-214.

5 Moon HJ, Son E, Kim EK, Yoon JH, Kwak JY. The diagnostic values of ultrasound and ultrasound-guided fine needle aspiration in subcentimeter-sized thyroid nodules. Ann Surg Oncol. 2012 Jan; 19(1):52-9.

6 Luo J, Zhang C, Huang F, Chen J, Sun Y, Xu $\mathrm{K}$, et al. Risk of malignancy in thyroid nodules: predictive value of puncture feeling of grittiness in the process of fine-needle aspiration. Sci Rep. 2017 Oct;7(1):13109.

7 Rosário PW, Calsolari MR. What is the best criterion for repetition of fine-needle aspiration in thyroid nodules with initially benign cytology? Thyroid. 2015 Oct;25(10):1115-20.

8 Poller DN, Baloch ZW, Fadda G, Johnson SJ, Bongiovanni M, Pontecorvi A, et al. Thyroid FNA: New classifications and new interpretations. Cancer Cytopathol. 2016;124(7):45766.
9 Cosgrove D, Barr R, Bojunga J, Cantisani V, Chammas MC, Dighe M, et al. WFUMB guidelines and recommendations on the clinical use of ultrasound elastography: part 4 Thyroid. Ultrasound Med Biol. 2017 Jan; 43(1):4-26.

10 Kameyama K, Ito K, Takami H. [Pathology of benign thyroid tumor]. Nihon Rinsho. 2007 Nov;65(11):1973-8.

11 Ruchala M, Szczepanek-Parulska E, Zybek A Moczko J, Czarnywojtek A, Kaminski G, et al. The role of sonoelastography in acute, subacute and chronic thyroiditis: a novel application of the method. Eur J Endocrinol. 2012 Mar;166(3):425-32.

12 Yang Z, Zhang H, Wang K, Cui G, Fu F. Assessment of diffuse thyroid disease by strain ratio in ultrasound elastography. Ultrasound Med Biol. 2015 Nov;41(11):2884-9.

13 Kakkos SK, Scopa CD, Chalmoukis AK, Karachalios DA, Spiliotis JD, Harkoftakis JG, et al. Relative risk of cancer in sonographically detected thyroid nodules with calcifications. J Clin Ultrasound. 2000 Sep;28(7):347-52.

14 Kobayashi K, Fujimoto T, Ota H, Hirokawa M, Yabuta T, Masuoka H, et al. Calcifications in thyroid tumors on ultrasonography: calcification types and relationship with histopathological type. Ultrasound Int Open. 2018 Apr;4(2):E45-51.

15 Haugen BR, Alexander EK, Bible KC, Doherty GM, Mandel SJ, Nikiforov YE, et al. 2015 American Thyroid Association Management Guidelines for Adult Patients with Thyroid Nodules and Differentiated Thyroid Cancer: The American Thyroid Association Guidelines Task Force on Thyroid Nodules and Differentiated Thyroid Cancer. Thyroid. 2016 Jan;26(1):1-133.

16 Gharib H, Papini E, Paschke R, Duick DS, Valcavi R, Hegedüs L, et al. American Association of Clinical Endocrinologists, Associazione Medici Endocrinologi, and European Thyroid Association medical guidelines for clinical practice for the diagnosis and management of thyroid nodules: executive summary of recommendations. J Endocrinol Invest. 2010;33(5 Suppl 1):51-6.
17 Smith-Bindman R, Lebda P, Feldstein VA, Sellami D, Goldstein RB, Brasic N, et al. Risk of thyroid cancer based on thyroid ultrasound imaging characteristics: results of a population-based study. JAMA Intern Med. 2013 Oct;173(19):1788-96.

18 Asteria C, Giovanardi A, Pizzocaro A, Cozzaglio L, Morabito A, Somalvico F, et al. USelastography in the differential diagnosis of benign and malignant thyroid nodules. Thyroid. 2008 May; 18(5):523-31.

19 Sun CY, Lei KR, Liu BJ, Bo XW, Li XL, He YP, et al. Virtual touch tissue imaging and quantification (VTIQ) in the evaluation of thyroid nodules: the associated factors leading to misdiagnosis. Sci Rep. 2017 Feb; 7:41958.

20 Aydoğan Bİ, Sahin M, Ceyhan K, Deniz O, Demir Ö, Emral R, et al. The influence of thyroid nodule size on the diagnostic efficacy and accuracy of ultrasound guided fine-needle aspiration cytology. Diagn Cytopathol. 2019 Jul; 47(7):682-7.

21 Yoon JH, Kwak JY, Moon HJ, Kim MJ, Kim EK. The diagnostic accuracy of ultrasoundguided fine-needle aspiration biopsy and the sonographic differences between benign and malignant thyroid nodules $3 \mathrm{~cm}$ or larger. Thyroid. 2011 Sep;21(9):993-1000.

22 Leenhardt L, Hejblum G, Franc B, Fediaevsky LD, Delbot T, Le Guillouzic D, et al. Indications and limits of ultrasound-guided cytology in the management of nonpalpable thyroid nodules. J Clin Endocrinol Metab. 1999 Jan; 84(1):24-8.

23 Lyu YJ, Shen F, Yan Y, Situ MZ, Wu WZ, Jiang GQ, et al. Ultrasound-guided fine-needle aspiration biopsy of thyroid nodules $<10 \mathrm{~mm}$ in the maximum diameter: does size matter? Cancer Manag Res. 2019;11:1231-6. 